\title{
Study of Myocardial Performance Index in Prediabetes and Its Correlation with Other Cardiovascular Risk Factors
}

\author{
T. K. Kambleㄹ, Ankita Kapse², Sunil Kumar³, Sourya Acharya ${ }^{4}$, Aiswarya Ghule ${ }^{5}$
}

\begin{abstract}
${ }^{1}$ Department of Internal Medicine, Datta Meghe Institute of Medical Sciences (Deemed to be University), Sawangi (Meghe) Wardha, Maharashtra, India. ${ }^{2}$ Department of Internal Medicine, Datta Meghe Institute of Medical Sciences (Deemed to be University), Sawangi (Meghe) Wardha, Maharashtra, India. ${ }^{3}$ Department of Internal Medicine, Datta Meghe Institute of Medical Sciences (Deemed to be University), Sawangi (Meghe) Wardha, Maharashtra,

India. ${ }^{4}$ Department of Internal Medicine, Datta Meghe Institute of Medical Sciences (Deemed to be University), Sawangi (Meghe) Wardha, Maharashtra, India. ${ }^{5}$ Department of Internal Medicine, Datta Meghe Institute of Medical Sciences (Deemed to be University), Sawangi (Meghe) Wardha, Maharashtra, India.
\end{abstract}

\section{ABSTRACT}

\section{BACKGROUND}

Myocardial performance index (MPI)/Tei index measures the LV systolic and LV diastolic function. Several studies have proved its reliability in evaluating the LV systolic and diastolic performance, with clear benefit over other old indices. It is also helpful in determining the prognosis of heart diseases, especially coronary artery disease. Diabetes affects the heart in several ways. The prevalence of coronary artery disease in a patient with diabetes is well known. The cardiac status in prediabetes is not studied extensively. Hence this study was undertaken to assess the myocardial function in prediabetes using MPI. We wanted to assess myocardial performance index (MPI) in prediabetes and correlate myocardial performance index with cardiovascular risk factors like waist hip ratio (WHR), fasting lipid profile (FLP) and body mass index (BMI).

\section{METHODS}

In this cross sectional study 200 prediabetics were enrolled as cases and 100 age and sex matched subjects were enrolled as controls. Thorough history, physical examination, biochemical investigations like FBS, PMBS, FLP and Echocardiography for myocardial performance index were done.

\section{RESULTS}

Body mass index (BMI), waist to hip ratio (WHR), total cholesterol, triglycerides, low density lipoprotein (LDL) and very low-density lipoprotein (VLDL) were significantly higher in prediabetes and high-density lipoprotein (HDL) were significantly lower in prediabetes in comparison to controls $(p=0.0001)$. Out of 200 prediabetic subjects, $23(11.5 \%)$ had abnormal MPI which was statistically significant in comparison with controls $(p=0.037)$ There was no correlation of abnormal myocardial performance index with cardiovascular risk factors like BMI, WHR and fasting lipid profile (FLP).

\section{CONCLUSIONS}

Prediabetes had abnormal MPI in comparison to controls. However, no correlation was found between cardiovascular risk factors and MPI.

\section{KEY WORDS}

Myocardial Performance Index (MPI), Echocardiography, Prediabetes
Corresponding Author:

Dr. Ankita Kapse,

Hinganghat, Wardha,

Maharashtra, India.

E-mail: ankita.kapse02@gmail.com

DOI: $10.14260 /$ jemds/2020/157

Financial or Other Competing Interests: None.

How to Cite This Article:

Kamble TK, Kapse A, Kumar S, et al. Study of myocardial performance index in prediabetes and its correlation with other cardiovascular risk factors. J. Evolution Med. Dent. Sci. 2020;9(10):721-725, DOI: $10.14260 /$ jemds/2020/157

Submission 17-09-2019,

Peer Review 08-02-2020,

Acceptance 14-02-2020,

Published 09-03-2020. 


\section{BACKGROUND}

According to International Diabetes Federation in 2017, 451 million people had Diabetes Mellitus in the world. Its prevalence is increasing rapidly and by 2045 , this number is estimated to be 693 million. There were 72.9 million patients who had diabetes mellitus in India in 2017 as per international diabetes federation diabetes atlas.(1) In 2012, 29.1 million Americans, i.e. 9.3\% of the population, had diabetes. In 2012, 86 million Americans of age 20 and older had prediabetes; this is raised from 79 million in 2010. Anjana RM et al in 2011 showed that, in India, prevalence of diabetes and prediabetes in 2011 was 62.4 and 77.2 million respectively.(2)

It had been observed that there is 6 fold increase risk of progression of IGT subjects to type 2 DM in comparison with that of normal glucose tolerance.(3) In 2011 Colagiuri S. et al showed that IFG and IGT individually had relative risks for progression to T2DM which was 4.7 -fold, as compared to normal fasting blood sugar and normal glucose tolerance. Furthermore, this risk is increased by 12 times when both IFG and IGT are present.(3) It has been seen that IGT is strongly associated with increase cardiovascular morbidities, whereas the association is relatively less for IFG. According to the guidelines issued by European Society for Cardiology (ESC) and European Association for the Study of Diabetes (EASD) 2007, prediabetes is more common in several conditions, such as: elderly, obesity especially central obesity, sedentary lifestyle, lack of healthy diet, family history of diabetes and hypertension. Garber AJ et al in 2008 stated that according to Pre-diabetes Consensus issued by American College of Endocrinology (ACE) and American Association of Clinical Endocrinology (AACE) in 2008, risk of development of diabetes and prediabetes are more common in the following conditions: family history, overweight and obesity, unhealthy life styles and hypertension.

In Type II DM there is dysfunction of the Beta-cell of the pancreas and insulin resistance. The onset of type 2 diabetes is gradual and patients progress through a state of prediabetes which is defined as one or more of the following: "Fasting blood glucose levels between $110 \mathrm{mg} / \mathrm{dl}$ to 125 $\mathrm{mg} / \mathrm{dl}$ (IFG) or/and two-hour plasma glucose level after 75-g OGTT between 140 to $199 \mathrm{mg} / \mathrm{dL}$ ". Prediabetes over a time progresses to diabetes which is a serious health problem in the modern population. There is increasing importance of the fact that modifications in the sedentary lifestyle are very helpful to delay the onset of pre-diabetes or it's progression to diabetes mellitus type-2.(2) There are many clinical trials that have shown that, moderate lifestyle modifications delay the onset of diabetes mellitus. The delay is by an average of 11 years and the occurrence of new-onset diabetes mellitus can be reduced at least by $20 \%$.(2) $^{2}$

Among populations with diabetes mellitus type II, leading cause of mortality is cardiovascular disease and there is evidence that at the time of diagnosis of diabetes mellitus, cardiac damage is often present, and people with hyperglycemia even when the value is less to call it as a diabetes, are known to be at a high risk for developing cardiovascular morbidities. The risk of developing cardiovascular disease when compared with normoglycemic subjects is increased by approximately $20 \%$. The prevalence of IFG increases with age. IFG is found to be more common in males than in females. Prediabetes goes overlooked due to the lack of awareness about prediabetes and lack of the screening methods of diabetes.(4) Even after excluding coronary artery disease and hypertension, DM is known to cause dysfunction of the left ventricle.(4) The association between left ventricular function and prediabetes has not been investigated enough and so we decided to investigate further about left ventricular dysfunction by myocardial performance index being the most accurate method, in prediabetes.

In 1995, TeiChuwa published an index known as myocardial performance index (MPI) which was named as the Tei index. This index evaluates both the functions of the heart i.e. the LV systolic function and LV diastolic function. This index is known to be a reflection of the morbity and mortality in cardiovascular disease. MPI is of a narrow range in healthy individuals. Traces of the aortic and mitral cardiac output by using Doppler are used to determine the myocardial performance index. This measurement is not affected by the heart rate, ventricular structure, afterload or age, thus suggesting lesser confounding factors. Hence this index was found to be reliable in evaluating the LV systolic and diastolic performance, with clear benefit over older established indices and for evaluating the prognosis in many heart diseases.(5)

MPI is more accurate because it is non-invasive, easy to perform. MPI has been used widely to assess quantitatively the performance of myocardium. It is more useful to assess the overall cardiac function of both the ventricles than systolic or diastolic function.(5) The MPI has a prognostic value in patients with risk factors for any cardiac disease. There is also prognostic value in patients with cardiac amyloidosis or myocardial infarction. However, there is little research on the risk factors for new- onset diabetes when coronary artery disease is not present. The MPI has been studied in many cardiac diseases, including diabetes, myocardial infarction, hypertension, and heart failure, and has a strong predictive relationship with morbidity and mortality.(6) Even though several groups have investigated the causes of left ventricular dysfunction in diabetes, very less studies have investigated the effects in prediabetes, the precursor of type $2 \mathrm{DM}$, on LV function. Hence, this study was undertaken to assess left ventricular function by myocardial performance index in subjects with prediabetes.

\section{METHODS}

This is a cross sectional study carried out in the Department of Medicine, Jawaharlal Nehru Medical College (JNMC), Sawangi, Wardha, Maharashtra. This college is attached to Acharya Vinobha Bhave Rural Hospital (AVBRH). This cross sectional study included 200 cases (prediabetic subjects) and 100 controls (age and sex matched asymptomatic individuals) from September 2016 to September 2018 after clearance from the institutional ethics committee. (IEC/201617/4072). The sample size was taken based on the convenience of the study. 


\section{Inclusion Criteria (Cases)}

All cases coming to OPD and IPD comprising the age group of 18 to 60 years, fulfilling WHO criteria. WHO Criteria for diagnosis of prediabetes was the criteria chosen for the study which stated that: "Fasting blood glucose level is between $110 \mathrm{mg} / \mathrm{dl}$ to $125 \mathrm{mg} / \mathrm{dl}$ (IFG) or/and Two-hour plasma glucose level after 75-g OGTT is between 140 to 199 $\mathrm{mg} / \mathrm{dl}$ ".(2) This criteria was stated by Buysschaert \& Bergman in 2009). Controls were healthy, asymptomatic, age and sex matched.

\section{Exclusion Criteria (Cases)}

Cases of Hypertension, diabetes mellitus, chronic corpulmonale, cardiomyopathy, ischemic heart disease, congenital heart disease, chronic alcoholism, chronic smokers and valvular heart disease were excluded from the study.

\section{Evaluation}

In all subjects, detail present history, past history, family history and personal history were collected. Thorough physical examination was done. Anthropometric measurements, investigations and echocardiography findings were as follows. Anthropometric features were measured thoroughly. They were weight, height, BMI, waist circumference (WC), hip circumference (HC), waist hip ratio (WHR). They were measured by standard methods. Biochemistry investigations including fasting blood sugar (FBS), post meal blood sugar (PMBS), serum total cholesterol (TC), triglyceride (TG), high-density lipoprotein (HDL), and low-density lipoprotein (LDL) and very low-density lipoprotein (VLDL) were done.

\section{Echocardiographic Examination}

The study was done on Philips Echocardiography Machine, (HD $11 \mathrm{XE}$ ) with standard 2-4 megahertz multi frequency probe. The myocardial performance index was calculated from the ratio of time intervals. These were derived with the help of pulsed Doppler echocardiography. The sample volume at the tips of the mitral valve leaflets, in the apical 4chamber view was located for measurement of intraventricular relaxation time (IVRT) and the intraventricular contraction time (IVCT). For measuring LV ejection time (b) the sample volume was then placed in the LV outflow tract, just below the aortic valve by the apical 5 -chamber view. The myocardial performance index (MPI) is defined as the sum of isovolumic contraction time plus isovolumic relaxation time and then divided by ejection time. In adult values of myocardial performance index greater than 0.40 are considered as abnormal.

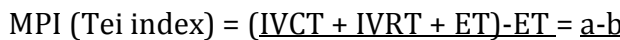

ET $b$

\section{Statistical Analysis}

Statistical analysis was done by using descriptive and inferential statistics. The chi-square test and Pearson's correlation coefficient were the tests used. The software used in the analysis were SPSS 22.0 version and Graph Pad Prism 6.0 version. $\mathrm{p}<0.05$ was considered as the level of significance. $\mathrm{P}$ value is the result of the $\mathrm{Z}$ test which shows the difference between two means, which is the test of significance.

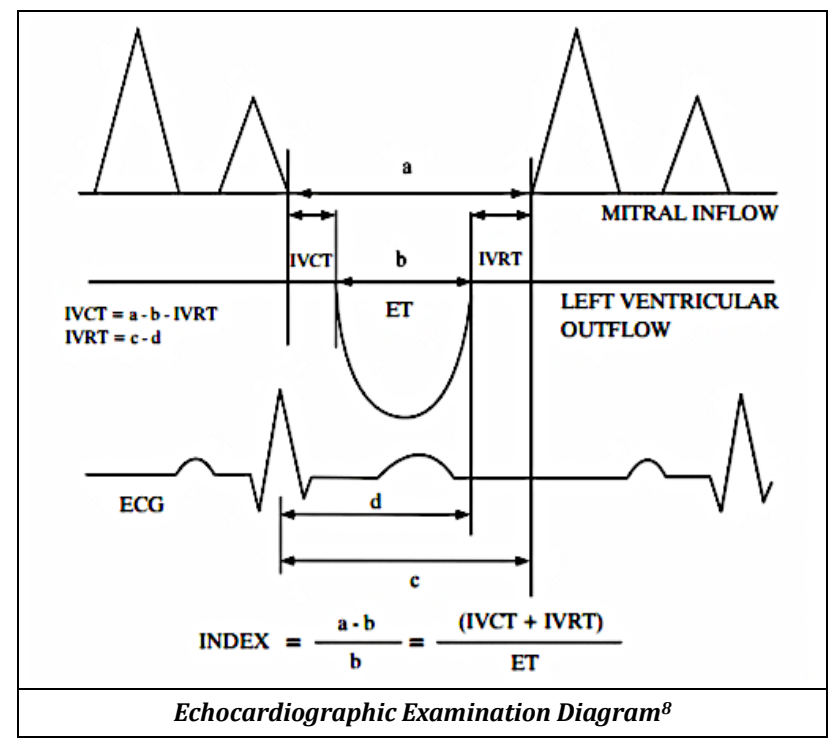

\section{RESULTS}

Out of 200 cases, $23(11.50 \%)$ cases were having high myocardial performance index i.e. $>0.40$. Out of 100 controls, $4(4 \%)$ control were having high myocardial performance index i.e. $>0.40$. Mean value of myocardial performance index for case was 0.387 . The standard deviation was 0.017 . Mean value of myocardial performance index for control was 0.381 with a standard deviation of 0.017 . Myocardial performance index was higher for cases than controls. Chi-square value was 4.38. $\mathrm{p}$-value was $0.049(\mathrm{p}<0.05)$ i.e. significant.

\begin{tabular}{|c|c|c|c|c|c|}
\hline \multirow{2}{*}{ Baseline Characteristics } & \multicolumn{2}{|c|}{ Cases } & \multicolumn{2}{|c|}{ Controls } & \multirow[t]{2}{*}{$\mathbf{p}$} \\
\hline & Mean & SD & Mean & SD & \\
\hline Age(yrs.) & 41.85 & 9.72 & 42.55 & 10.68 & $0.98 \mathrm{NS}$ \\
\hline \multicolumn{5}{|c|}{ Gender } & \\
\hline Male & 115 & 57.5 & 57 & 57 & $0.93 \mathrm{NS}$ \\
\hline Female & 85 & 42.5 & 43 & 43 & $0.50 \mathrm{NS}$ \\
\hline BMI (body mass index) & 24.92 & 3.39 & 22.08 & 2.35 & $0.0001 \mathrm{~S}$ \\
\hline WC( waist circumference) & 91.94 & 6.57 & 82.46 & 6.32 & \\
\hline HC( hip circumference) & 97.00 & 5.97 & 97.00 & 5.71 & \\
\hline \multicolumn{6}{|l|}{ WC/HC (waist hip ratio) } \\
\hline Male & 0.97 & 0.07 & 0.86 & 0.06 & $0.0001 \mathrm{~S}$ \\
\hline Female & 0.91 & 0.06 & 0.82 & 0.06 & $0.0001 \mathrm{~S}$ \\
\hline SBP (systolic blood pressure) & 125.82 & 8.65 & 125.06 & 9.33 & $0.03 \mathrm{NS}$ \\
\hline DBP (Diastolic Blood Pressure) & 80.72 & 10.46 & 80.40 & 6.31 & $0.15 \mathrm{NS}$ \\
\hline FBS ( Fasting Blood Sugar) & 118.15 & 5.22 & 94.42 & 9.84 & \\
\hline $\begin{array}{c}\text { OGTT (Oral Glucose Tolerance } \\
\text { Test) }\end{array}$ & 158.02 & 16.98 & 121.11 & 18.62 & \\
\hline TC ( Total Cholesterol) & 182.05 & 35.06 & 161.29 & 23.64 & $0.0001 \mathrm{~S}$ \\
\hline TG (total triglyceride) & 141.41 & 47.44 & 120.07 & 30.99 & $0.0001 \mathrm{~S}$ \\
\hline LDL (low density lipoprotein) & 116.60 & 33.98 & 89.46 & 34.43 & $0.0001 \mathrm{~S}$ \\
\hline \multicolumn{6}{|l|}{ HDL (high density lipoprotein) } \\
\hline Male & 40.77 & 13.05 & 52.57 & 15.85 & $0.0001 \mathrm{~S}$ \\
\hline Female & 40.77 & 13.05 & 49.89 & 14.89 & $0.008 \mathrm{~S}$ \\
\hline $\begin{array}{l}\text { VLDL (very low density } \\
\text { lipoprotein) }\end{array}$ & 28.76 & 10.83 & 25.02 & 8.73 & $0.0001 \mathrm{~S}$ \\
\hline $\begin{array}{l}\text { MPI (myocardial performance } \\
\text { index) }\end{array}$ & 0.387 & 0.017 & 0.381 & 0.017 & $0.0001 \mathrm{~S}$ \\
\hline (1) & & & & & \\
\hline
\end{tabular}

Table 1 shows cases and controls were age and sex matched ( $p$-not significant). BMI, WHR, TC, TG, LDL, VLDL, MPI were significantly higher in prediabetes. While, HDL was significantly lower in pre-diabetes as compared to controls. (p- significant).(1) 


\begin{tabular}{|c|c|c|c|c|c|}
\hline \multirow{2}{*}{ MPI } & \multicolumn{2}{|c|}{ Cases } & \multicolumn{2}{|c|}{ Control } & \multirow{2}{*}{$\chi 2$-Value } \\
\hline & $\mathbf{n}=\mathbf{2 0 0}$ & $\%$ & $\mathrm{n}=\mathbf{1 0 0}$ & $\%$ & \\
\hline$\leq 0.40$ & 177 & 88.5 & 96 & 96 & \multirow{5}{*}{$\begin{array}{c}4.38 \\
\mathrm{p}=0.037 \\
\text { significant }\end{array}$} \\
\hline$>0.40$ & 23 & 11.5 & 4 & 4 & \\
\hline Total & 200 & 100 & 100 & 100 & \\
\hline Mean \pm SD & \multicolumn{2}{|c|}{$0.387 \pm 0.017$} & \multicolumn{2}{|c|}{$0.381 \pm 0.017$} & \\
\hline Range & \multicolumn{2}{|c|}{$0.33-0.46$} & \multicolumn{2}{|c|}{$0.33-0.44$} & \\
\hline \multicolumn{6}{|c|}{ Table 2. Distribution of Cases and Controls According to MPI } \\
\hline able 2 shov & ras sig & Vy his & ses in & isor & lo \\
\hline
\end{tabular}

\begin{tabular}{|c|c|c|}
\hline Parameters & Correlation 'r' & p-Value \\
\hline MPI & - & - \\
\hline BMI & 0.056 & $0.431, \mathrm{NS}$ \\
\hline WC & -0.087 & $0.220, \mathrm{NS}$ \\
\hline HC & 0.010 & $0.886, \mathrm{NS}$ \\
\hline WC/HC & -0.091 & $0.200, \mathrm{NS}$ \\
\hline SBP & 0.084 & $0.237, \mathrm{NS}$ \\
\hline DBP & 0.006 & $0.932, \mathrm{NS}$ \\
\hline FBS & 0.078 & $0.273, \mathrm{NS}$ \\
\hline OGTT & 0.126 & $0.076, \mathrm{NS}$ \\
\hline TC & -0.123 & $0.083, \mathrm{NS}$ \\
\hline TG & -0.058 & $0.418, \mathrm{NS}$ \\
\hline HDL & 0.039 & $0.582, \mathrm{NS}$ \\
\hline VLDL & 0.073 & $0.305, \mathrm{NS}$ \\
\hline LDL & 0.088 & $0.216, \mathrm{NS}$ \\
\hline \multicolumn{2}{|c|}{ Table 3. Correlation of MPI with Different } \\
\hline \multicolumn{2}{|c|}{ Cardiovascular Risk Factors $\left.{ }^{1}\right)$} \\
\hline $\begin{array}{l}\text { Pearson's correlation coefficient analysis showed that there is no correlation of } \\
\text { cardiovascular risk factors with MPI in prediabetes. }\end{array}$ \\
\hline
\end{tabular}

\section{DISCUSSION}

MPI depends on impairment in glucose metabolism. Certain end products of glycosylation are formed due to hyperglycemia which cause damage to cardiac muscle fibres. This further increases fibrosis as a result of oxidative stress and inflammation. In a heart compromised by diabetes mellitus the following changes might occur. These are ventricular hypertrophy, remodelling and fibrosis of the heart tissue, changes in the blood vessels and insulin resistance. They are the most important changes that may affect the myocardial function. (5) Hyperglycemia may also stimulate apoptosis and myocyte necrosis.(7) This results in the loss of cardiomyocytes which may impair the contractility of the myocardium and lead to systolic dysfunction. Several molecular signalling pathways contribute to the dysfunctioning of the cardiac myocyte in patients with prediabetes. Therefore, it has been found that people having prediabetes may have increased MPI due to prolonged exposure to increased levels of glucose. Additionally, free fatty acids, that can cause lipotoxicity and damage are also increased in the circulation. ${ }^{(5)}$

A recent cross-sectional study by Stahrenberg R, Edelmann F, et al reported that the prevalence of $\mathrm{LV}$ dysfunction by measuring myocardial performance index increased in patients with prediabetes and its severity was directly proportional to the severity of impairment of glucose metabolism. Several studies have shown that, in 2D echocardiography, the diastolic function of the heart is affected even before the dysfunction of the left ventricular systolic function. This is an early finding in 2D echo in a patient with diabetes.(5) It was also observed that this dysfunction is often found even before the development of diabetes mellitus, thus suggesting that this dysfunction is a co-existing condition and not a necessary complication of diabetes. (5)

In our study BMI was statistically higher in prediabetes in comparison to age and sex matched controls. The $P$ value was significant. $(\mathrm{p}<0.05, \mathrm{~S})$ Similarly, Murat Akçay et al. reported BMI was statistically higher in prediabetes in comparison to age and sex matched controls. (8) In our study waist hip ratio was higher in prediabetes when compared to age and sex matched controls. However, Murat Akçay et al reported that mean waist hip ratio for cases and controls were almost similar. Difference in two groups was found to be statistically insignificant.(8) We have also found that total cholesterol, triglycerides, LDL and VLDL were significantly higher in prediabetes and high density lipoprotein were significantly lower in prediabetes as compared to controls $(\mathrm{p}=0.0001)$. Same observation was seen by Murat Akçay et al.(8) Myocardial performance index in cases was higher than controls. Difference in MPI in cases and controls was found to be statistically significant. However, Murat Akçay1 et al (2016) observed that mean value of myocardial performance index in prediabetes was $0.51 \pm 0.09$. Mean value of myocardial performance index in controls was $0.48 \pm 0.08$. Myocardial performance index in cases was higher than controls. Difference in two groups was not found to be statistically significant.

There was no significant co-relation of MPI with cardiovascular risk factors like body mass index, waist-hip ratio, and total cholesterol, triglyceride, high density lipoprotein, low density lipoprotein and very low-density lipoprotein. However, there are no studies available on correlation between MPI in prediabetes and its correlation with cardiovascular risk factors.

\section{Limitations}

Our study has been of a relatively small sample size. This study was conducted using a hospital-based sample. Thus we will need further research in order to confirm whether these conclusions can be generalized in the population.

\section{CONCLUSIONS}

Body mass index and waist to hip ratio were (statistically) significantly higher in prediabetes as compared to controls. Prediabetes had (statistically) significantly impaired lipid profile as compared to controls. Myocardial performance index (MPI) was (statistically) significantly higher in prediabetes as compared to controls. Pearson's Correlation Coefficient analysis showed that there was no significant corelation of MPI with cardiovascular risk factors.

\section{REFERENCES}

[1] Buysschaert M, Bergman M. Definition of pre-diabetes. Med Clin N Am 2011;95(2):289-97.

[2] Kenealy T, Elley CR, Arrol B. Screening for diabetes and pre-diabetes. Lancet 2007;370(9603):1888-9. 
[3] Lakoumentas JA, Panou FK, Kotseroglou VK, et al. The Tei index of myocardial performance: applications in cardiology. Hellenic J Cardiol 2005;46(1):52-8.

[4] Tei C, Dujardin KS, Hodge DO, et al. Doppler echocardiographic index for assessment of global right ventricular function. $\mathrm{J}$ Am Soc Echocardiogr 1996;9(6):838-47.

[5] Stahrenberg R, Edelmann F, Mende M, et al. Association of glucose metabolism with diastolic function along the diabetic continuum. Diabetologia 2010;53(7):1331-40.

[6] Paczek A, Gardin JM, Hardin JM, et al. Comparison of Mmode and two-dimensional echocardiographic algorithms used to estimate left ventricular mass: the coronary artery risk development in young adults. J Am Soc Echocardiogr 1995;8(6):780-92.
[7] Cai L, Li W, Wang G, et al. Hyperglycemia-induced apoptosis in mouse myocardium: mitochondrial cytochrome C-mediated caspase-3 activation pathway. Diabetes 2002;51(6):1938-48.

[8] Akcay M, Asian AN, Kasapkara HA, et al. Assessment of the left ventricular function in normotensive prediabetics: a tissue Doppler echocardiography study. Arch Endocrinol Metab 2016;60(4):341-7. 